\title{
Expression of microRNA-21 in osteoporotic patients and its involvement in the regulation of osteogenic differentiation
}

\author{
ZHONGFU ZHAO ${ }^{1}$, XIAOGUANG LI ${ }^{1}$, DEXUN ZOU ${ }^{1}$, YONGYUN LIAN $^{2}$, SHAOHUA TIAN ${ }^{3}$ and ZHI DOU \\ ${ }^{1}$ Department of Orthopaedic Surgery, The Second Affiliated Hospital of Qiqihar Medical University, Qiqihar, \\ Heilongjiang 161006; ${ }^{2}$ Department of Orthopaedics, The Fourth Affiliated Hospital of Harbin Medical University, Harbin, \\ Heilongjiang 150001; ${ }^{3}$ Department of Orthopaedics, The Third Affiliated Hospital of Qiqihar Medical University, \\ Qiqihar, Heilongjiang 161000; ${ }^{4}$ Department of Orthopaedics (II), The First Hospital of Qiqihar City, \\ Qiqihar, Heilongjiang 161005, P.R. China
}

Received May 14, 2018; Accepted November 9, 2018

\section{DOI: $10.3892 /$ etm.2018.6998}

\begin{abstract}
Expression of microRNA-21 in bone tissue and serum of patients with osteoporosis (OP) and its involvement in the regulation of osteogenic differentiation of rat bone marrow mesenchymal stem cells (BMSCs) were investigated. Bone tissue and serum were collected from 48 patients with OP and 48 normal subjects. Reverse transcription-quantitative polymerase chain reaction (RT-qPCR) was used to detect the expression of six microRNAs. Among these microRNAs, the expression level of microRNA-21 in bone tissue and serum of OP patients was the lowest. In addition, BMSCs of SD rats were isolated and cultured. Subculture was performed 3 times, transfection of microRNA-21 was performed and osteogenic differentiation was induced. Control group [negative control (NC)] was transfected with microRNA-21 mimics followed by osteogenic induction. Experimental groups were transfected with microRNA-21 analogue (mimics) and microRNA-21 inhibitor (inhibitor) followed by osteogenic induction. Ten days after osteogenic induction, alkaline phosphatase (ALP) staining and alizarin red staining were performed to measure the mineralized stained area and the number of mineralized nodules in each treatment group. RT-qPCR was used to detect the expression of osteogenic genes in each group of cells. RT-qPCR results showed that microRNA-21 expression was lower in bone tissue and serum of patients with OP than that of normal subjects. Moreover, compared with control group, BMSCs showed increased stained mineralized areas, deeper color and increased number of mineralized nodules. In addition, increased mRNA expression of osteogenic genes was evident after microRNA-21 mimics
\end{abstract}

Correspondence to: Dr Zhongfu Zhao, Department of Orthopaedic Surgery, The Second Affiliated Hospital of Qiqihar Medical University, 37 Zhonghua West Road, Qiqihar, Heilongjiang 161006, P.R. China

E-mail: fzn4g8@163.com

Key words: bone marrow mesenchymal stem cells, microRNA-21, osteogenic differentiation, osteoporosis transfection and osteogenic induction $(\mathrm{p}<0.05)$. Compared with control group, BMSCs showed decreased stained mineralized areas, lighter color, decreased number of mineralized nodules, and decreased mRNA expression of osteogenic genes after microRNA-21 inhibitor transfection and osteogenic induction $(\mathrm{p}<0.05)$. MicroRNA-21 is expressed at low level in bone tissue and serum in patients with OP, and microRNA-21 can promote osteogenic differentiation of BMSCs. Our study provided theoretical basis for drug treatment of OP.

\section{Introduction}

With the growth of aging population in China, osteoporosis (OP) has become one of the most common chronic diseases (1). OP is a metabolic orthopedic disease characterized by decreased bone mass, reduced bone density, and degraded bone structure. OP is mostly present in elderly and postmenopausal women, and the incidence is higher in females than in males $(2,3)$. In patients with OP, the balance of bone metabolism in the body is broken and bone absorption is much greater than bone formation. Patient experience bone pain, kyphosis, shortened body, decreased respiratory function, and fractures, which seriously harm the whole body (4). At present, calcium, physical activity and bone resorption inhibitors are widely used in the treatment of OP, but these measures can only alleviate the patient's symptoms, and cannot prevent the occurrence of the disease $(5,6)$. Studies have shown that bone marrow mesenchymal stem cells (BMSCs), as the main source of osteoblasts in humans, have multi-directional differentiation potential and can differentiate into osteoblasts, chondrocytes and adipocytes under certain conditions. Therefore, BMSCs play a very important role in the treatment of OP $(7,8)$.

MicroRNAs are a class of non-coding RNAs of 22 nucleotides in length that are widely conserved across species. MicroRNAs degrade mRNA and inhibit translation by specifically binding to the 3'-untranslated region (3'-UTR) of messenger RNA of the target gene $(9,10)$. MicroRNAs are involved in the transcriptional regulation of many cells or other basic physiological activities such as cell survival, cell proliferation, and apoptosis (11). MicroRNA-21 is differentially expressed in cardiovascular diseases, cancer, renal and digestive system 
diseases, and thus participates in their pathophysiological process (12). However, whether microRNA-21 is differentially expressed in patients with OP and whether it can regulate the differentiation of BMSCs into osteoblasts, thereby affecting the balance of bone metabolism, is still unknown. Therefore, this study investigated the expression of microRNA-21 in OP patients and its influence on the osteogenic differentiation of BMSCs.

\section{Materials and methods}

Materials. Fetal bovine serum and carbon dioxide incubator was obtained from Thermo Fisher Scientific, Inc. (Waltham, MA, USA), and inverted optical microscope from Olympus Corp. (Tokyo, Japan). SD rats were purchased from the Animal Experimental Center of the Second Affiliated Hospital of Harbin Medical University (Harbin, China), and microRNA-21 mimics, inhibitors and negative controls (NCs) from Invitrogen (Invitrogen; Thermo Fisher Scientific). DMEM/F12 culture medium was provided by Wisent Biotechnology (Nanjing, China). Trypsin digestion solution was obtained from Beyotime Institute of Biotechnology (Haimen, China). Complete culture medium (F12 medium $+10 \%$ fetal bovine serum $+1 \%$ cyanine-streptomycin), osteogenic medium $(\mathrm{F} 12$ medium $+10 \%$ fetal bovine serum $+10 \mathrm{mM} \beta$-sodium glycerophosphate $+0.05 \mathrm{mM}$ vitamin $\mathrm{C}+10 \mathrm{mM}$ dexamethasone $+1 \%$ cyanine-streptomycin), alkaline phosphatase (ALP) staining solution $(10 \mathrm{ml}$ distilled water $+5 \mathrm{ml} \mathrm{3 \%} \beta$-sodium glycerophosphate $+5 \mathrm{ml} 2 \%$ pentobarbital $+10 \mathrm{ml} 2 \%$ calcium chloride $+1 \mathrm{ml} 2 \%$ magnesium sulfate, $\mathrm{pH}$ 9.4) were prepared by the authors of this study. Alizarin red staining medium (Cyagen Biosciences, Inc., Santa Clara, CA, USA), TRIzol (Sigma-Aldrich; Merck KGaA, St. Louis, MO, USA), reverse transcription-quantitative polymerase chain reaction (RT-qPCR) primers (Invitrogen; Thermo Fisher Scientific, Inc.), reverse transcription kit and reverse transcription-polymerase chain reaction (both from Applied Biosystems; Thermo Fisher Scientific, Inc., Foster City, CA, USA), cyan-streptomyces (Beyotime Institute of Biotechnology), RT-qPCR instrument and SYBR-Green (both from Roche Diagnostics GmbH, Mannheim, Germany), pipette (Thermo Fisher Scientific, Inc.) and RNase-free tips (Qiagen Sciences, Inc., Germantown, MD, USA) were used in our experiments. All other experimental reagents were purchased from Sigma-Aldrich (Sigma-Aldrich; Merck KGaA).

Collection of clinical samples and microRNA detection. During the period from June 2016 to December 2017, bone tissue was collected from patients with OP and normal subjects at the Department of Orthopaedic Surgery of the Second Affiliated Hospital of Qiqihar Medical University (Qiqihar, China). The study was approved by the Ethics Committee of the Second Affiliated Hospital of Qiqihar Medical University. Signed informed consents were obtained from the patients or the guardians.

Patients with diabetes mellitus, hyperprolactinemia, ovariectomy, liver cancer, and malabsorption syndrome affect bone metabolic disease were excluded. Bone tissues of $48 \mathrm{OP}$ patients were set as experimental group, and bone tissues of 48 normal subjects were set as control group. The expression of
microRNA-17, microRNA-20a, microRNA-21, microRNA-26a, microRNA-29b, and microRNA-106b was detected. Briefly, bone tissues were ground in liquid nitrogen, followed by addition of TRIzol to extract total RNA. Reverse transcription was performed to synthesize DNA according to the manufacturer's instructions. cDNA samples were stored at $-20^{\circ} \mathrm{C}$ before use. qPCR reaction system included $10 \mu \mathrm{l} \mathrm{SYBR-Green,} 7 \mu \mathrm{lddH_{2 }} \mathrm{O}$, $1 \mu \mathrm{l}$ upstream primers, $1 \mu \mathrm{l}$ downstream primers, and $1 \mu \mathrm{l} \mathrm{cDNA}$. qPCR reaction conditions were: $95^{\circ} \mathrm{C}$ for $30 \mathrm{sec}$, followed by 40 cycles of $95^{\circ} \mathrm{C}$ for $5 \mathrm{sec}, 60^{\circ} \mathrm{C}$ for $30 \mathrm{sec}$ and $72^{\circ} \mathrm{C}$ for $30 \mathrm{sec}$. The experiment was performed in triplicate, and the expression of each microRNA was normalized to U6 endogenous control using $2^{-\Delta \Delta \mathrm{Cq}}$ method (Table I) (13).

Primary culture and subculture of rat BMSCs. A total of 24 4-week-old SD female rats (70-90 g) were maintained under specific pathogen-free conditions $\left(22^{\circ} \mathrm{C}, 12\right.$-h light/12-h dark cycles, and 50-55\% humidity) and were used to isolate tibias and femurs under aseptic conditions. Bone tissues were cleaned and sterilized with $75 \%$ alcohol, and the epiphyses were cut off. A syringe $(1 \mathrm{ml})$ was used to inject complete culture fluid into the cavity to flush bone marrow, and this procedure was performed 3 times until the bone became transparent. The flushed bone marrow was mixed with $4 \mathrm{ml}$ of culture medium, inoculated into a $25-\mathrm{cm}^{2}$ perforated cell culture flask and cultured in an incubator $\left(37^{\circ} \mathrm{C}, 5 \% \mathrm{CO}_{2}\right)$. Medium was replaced every $48 \mathrm{~h}$. When cell confluence reached $80 \%$, digestion with trypsin and subculture were performed. Subculture was performed 3 times before subsequent experiments.

Cell transfection. Cell transfection was performed when confluence of rat BMSCs reached 50-60\%. Cells were starved for $2 \mathrm{~h}$ in serum-free Opti media. Lipofectamine 2000 was used to transfect $50 \mathrm{nM}$ microRNA-21 mimics/NC or $100 \mathrm{nM}$ microRNA-21 inhibitor/NC into cells. Cells were observed $6 \mathrm{~h}$ after transfection and the medium was replaced.

Osteogenic differentiation of rat BMSCs. Subculture was performed 3 times and BMSCs were collected. Osteogenic induction medium was added $24 \mathrm{~h}$ after transfection with microRNA-21 mimic, inhibitor and NC. Induction was performed for 10 days, medium was replaced with fresh osteogenic induction medium every 3 days, and each experiment was repeated 3 times.

Identification of osteogenic differentiation of BMSCs and comparison between groups. After transfection and osteogenic induction, cells were washed 3 times with PBS, followed by fixation in $4 \%$ paraformaldehyde at room temperature for 20 min. After that, incubation with ALP staining solution for $4 \mathrm{~h}$ and alizarin red staining solution for $30 \mathrm{~min}$ was performed. Cells were washed with PBS and observed under an inverted light microscope. Differentiation ability was determined according to the area and color of mineralized nodule.

Detection of the expression of osteogenesis-related genes. After transfection and osteogenic induction, RT-qPCR was used to detect the expression of intracellular osteogenic genes including ALP, osteocalcin (OCN), bone morphogenetic protein 2 (BMP2) and runt-related transcription 
Table I. qPCR primer sequences for microRNA detection.

\begin{tabular}{llc}
\hline Gene & \multicolumn{1}{c}{ Forward } & Reverse \\
\hline microRNA-21 & CGCCGTAGCTTATCAGACTG & CAGCCACAAAAGAGCACAAT \\
U6 & GCTTCGGCAGCACATATACTAAAAT & CGCTTCACGAATTTGCGTGTCAT
\end{tabular}

Table II. qPCR primer sequences for the detection of the expression of osteogenesis-related genes.

\begin{tabular}{lll}
\hline Gene & \multicolumn{1}{c}{ Forward } & Reverse \\
\hline ALP & ACAACCTGACTGACCCTTCG & TCATGATGTCCGTGGTCAAT \\
OCN & GGACCATCTTTCTGCTCACTC & CTGCTTGGACATGAAGGCTT \\
BMP2 & CCTTGCTGACCACCTGAACT & AACATGGAGATTGCGCTGA \\
Runx2 & AGAAGGCACAGACAGAAGCTT-GA & AGGAATGCGCCCTAAATCACT \\
GAPDH & CATCACTGCCACCCAGAAGAC & CCAGTGAGCTTCCCGTTCAG \\
\hline
\end{tabular}

ALP, alkaline phosphatase; OCN, osteocalcin; BMP2, bone morphogenetic protein 2; Runx2, runt-related transcription factor 2; GAPDH, glyceraldehyde-3-phosphate dehydrogenase.

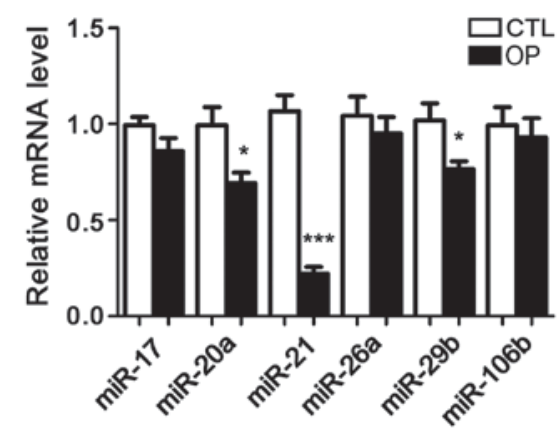

Figure 1. MicroRNA expression levels in bone tissues of patients and controls. In patients, microRNA-21 had the lowest expression level. ${ }^{*} \mathrm{P}<0.05$ and **** $\mathrm{p}<0.001$.

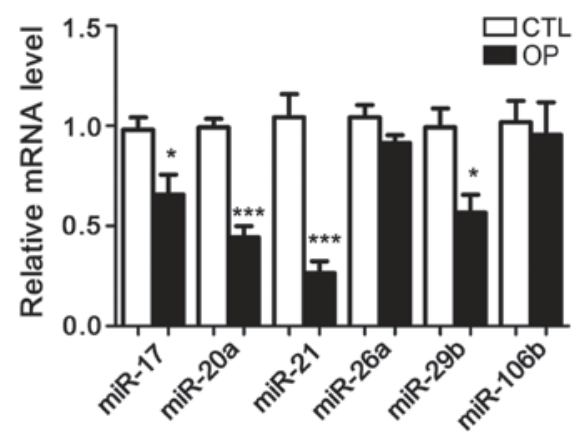

Figure 2. MicroRNA expression levels in serum of patients and controls. In patients, microRNA-21 had the lowest expression level. ${ }^{*} \mathrm{P}<0.05$ and ${ }^{* * * *} \mathrm{p}<0.001$.

factor 2 (Runx2) using methods given in Collection of clinical samples and microRNA detection' (Table II).

Statistical analysis. All data were expressed as mean \pm SEM and were statistically processed using GraphPad software (GraphPad Software, Inc., La Jolla, CA, USA). t-test

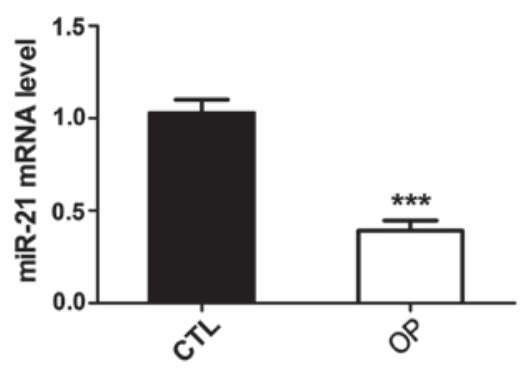

Figure 3. MicroRNA-21 expression in BMSCs of osteoporotic rats. Compared with the control group, the expression level of microRNA-21 in BMSCs of osteoporotic rats was significantly reduced. ${ }^{* * *} \mathrm{P}<0.001$. BMSCs, bone marrow mesenchymal stem cells.

was used for comparison between two groups. $\mathrm{P}<0.05, \mathrm{p}<0.01$ and $\mathrm{p}<0.001$ were considered to be statistically significant, as indicated in the figures.

\section{Results}

Detection of microRNA expression in bone tissues and serum samples. Following extraction of total RNA from bone tissue of patients and healthy controls, the expression of six microRNAs including microRNA-17, microRNA-20a, microRNA-21, microRNA-26a, microRNA-29b, and microRNA-106b was detected. It has been reported that these six microRNAs are related to OP in mice, but no studies have shown whether they are differentially expressed in patients with OP. The results showed that compared with control group, the expression of microRNA-21 in patients with OP was significantly reduced. In addition, the serum levels of microRNA-21 were also significantly lower in patients than in controls. Therefore, microRNA-21 was selected as the object of our study (Figs. 1 and 2).

Detection of microRNA-21 expression in BMSCs of osteoporotic rats. BMSCs of normal and osteoporotic rats were 

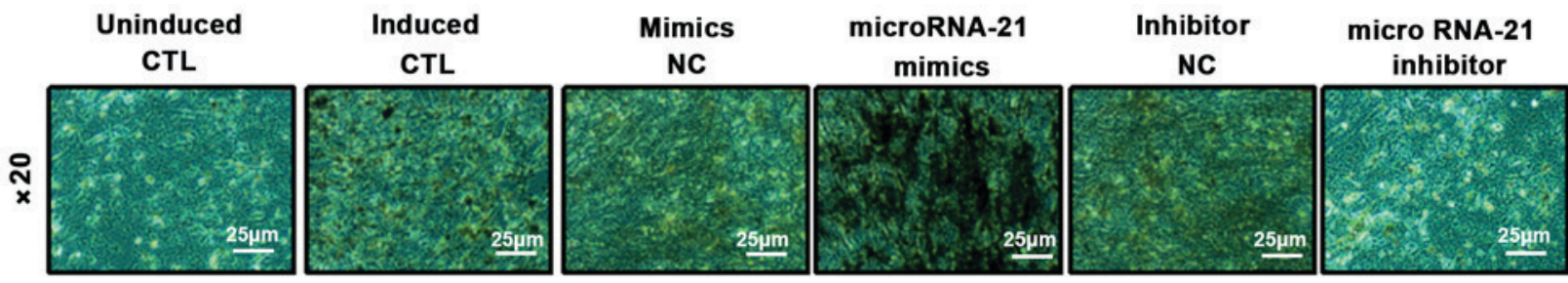

Figure 4. ALP staining of osteogenic differentiation of BMSCs. ALP, alkaline phosphatase; BMSCs, bone marrow mesenchymal stem cells.
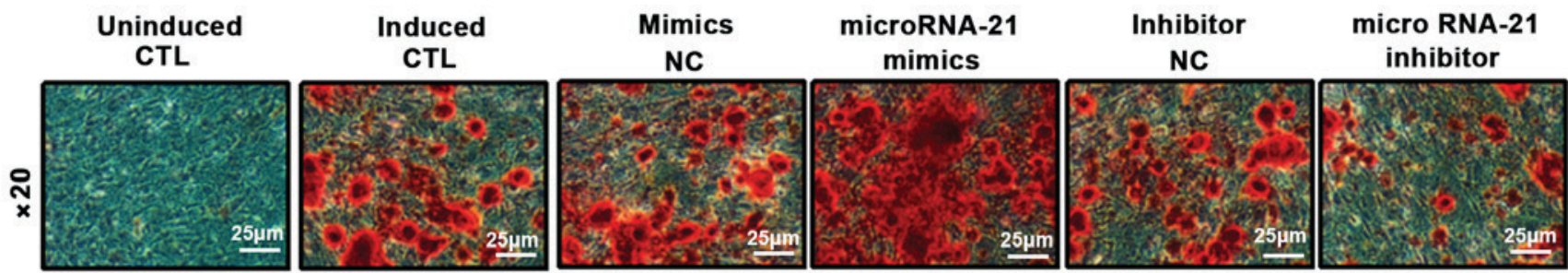

Figure 5. ARS staining of osteogenic differentiation of BMSCs. ARS, alizarin red S; BMSCs, bone marrow mesenchymal stem cells.
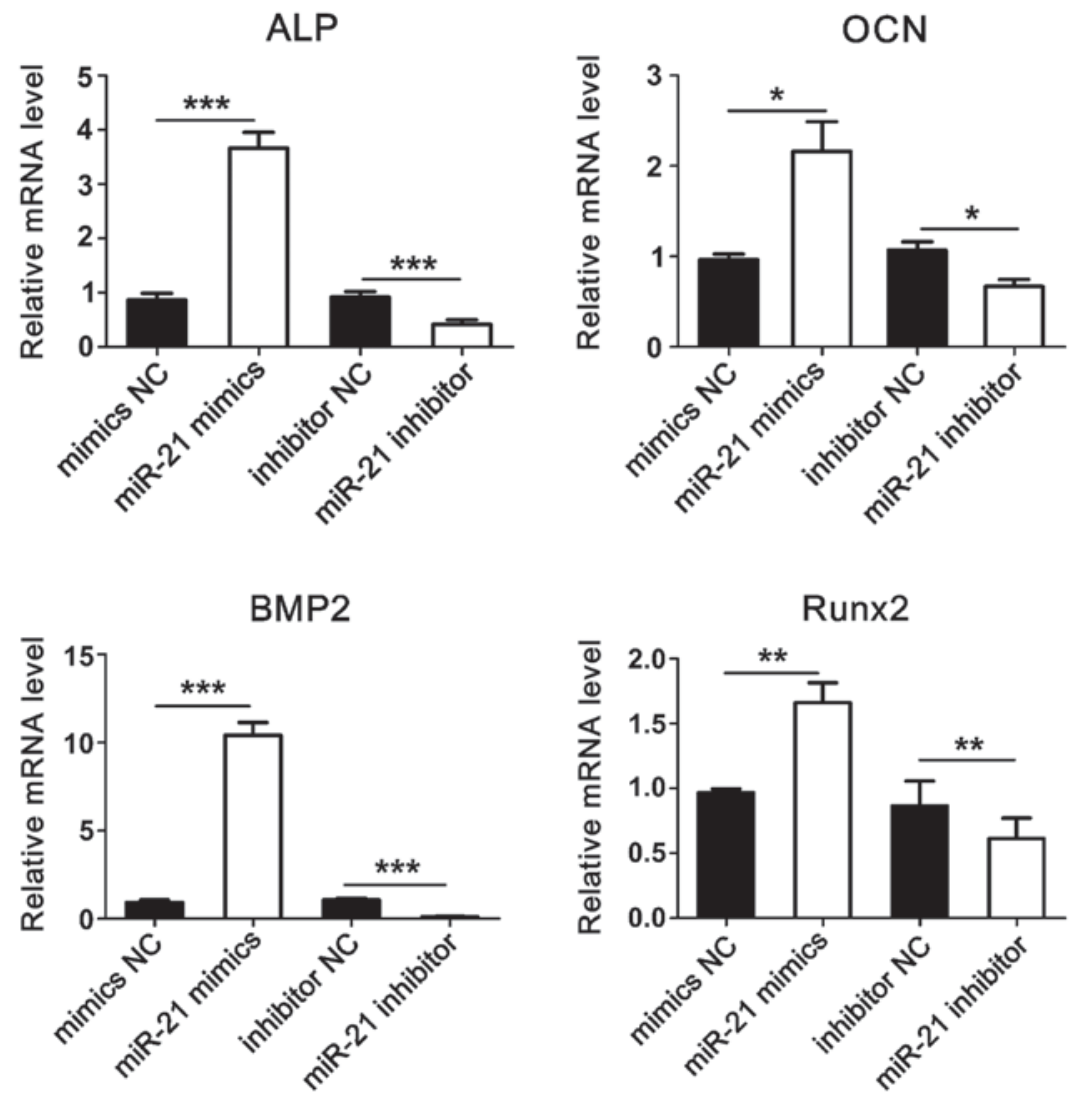

Figure 6. Expression levels of osteogenesis-related genes. ${ }^{*} \mathrm{P}<0.05,{ }^{* *} \mathrm{p}<0.01$ and ${ }^{* * *} \mathrm{p}<0.001$.

isolated and total RNA was extracted to detect the expression of microRNA-21. Results showed that compared with the control group, the expression level of microRNA-21 in BMSCs of osteoporotic rats was significantly reduced (Fig. 3).

Identification and comparison of osteogenic differentiation. BMSCs were transfected with microRNA-21 mimics, inhibitors and their corresponding NCs. ALP staining and alizarin red staining were performed 10 days after osteogenic induction to detect the area and number of nodules in the mineralized area, and calcium deposition was assessed. ALP results showed that compared with the control group, mineralized nodule staining in the microRNA-21 mimics group was significantly deepened, and the area of mineralized nodules was increased. However, the mineralized nodules in microRNA-21 inhibitor group became lighter and the area of mineralized nodules 
decreased. Alizarin red S (ARS) results showed that compared with control group, orange-red color was significantly deepened and calcium deposition was significantly increased in microRNA-21 mimics group. However, ARS staining in microRNA-21 inhibitor group was lighter and calcium deposition was significantly reduced. These results indicate that microRNA-21 has the ability to promote the differentiation of BMSCs into osteoblasts (Figs. 4 and 5).

Expression of osteogenesis-related genes. After transfection and osteogenic induction, total RNA was extracted and RT-qPCR was used to detect the expression of ALP, OCN, BMP2, and Runx2 genes. Results showed that compared with the control group, the expression levels of osteogenesis-related genes were significantly increased in the microRNA-21 mimics group. Conversely, the expression levels of osteogenesis-related genes were significantly reduced in microRNA-21 inhibitor group (Fig. 6).

\section{Discussion}

OP is a systemic metabolic disease characterized by reduced bone mass, reduced bone density, and reduced bone microstructure $(14,15)$. Patients with OP have reduced trabecular number, increased brittleness of bones, and markedly decreased bone strength, resulting in increased fracture risk, which brings pain and financial burden to patients and their families (16). At present, incidence of OP is increasing year by year, and it has become one of the most common diseases in the world. Therefore, studies on OP have attracted increasing attention (17).

BMSCs are a type of adult stem cells that exist in the bone marrow and have self-renewal ability and multi-directional differentiation potential. Under certain induction conditions, they can differentiate into many kinds of cells, such as osteoblasts, adipocytes, chondrocytes, cardiomyocytes and nerve cells (18). The osteogenic potential of BMSCs plays an important role in the treatment of OP, bone fractures, bone necrosis, osteoarthritis and bone defects $(19,20)$. Therefore, to study the differentiation of BMSCs is of great significance for the treatment of OP.

An increasing number of studies have shown that microRNA can be used as a key regulator of the osteogenesis of BMSCs. For example, it has been reported that microRNA-320a can inhibit the differentiation of human BMSCs into osteoblasts (21). In another study, microRNA-196a was reported to promote osteogenic differentiation of human adipocytes and inhibited their proliferation (22). Recently, it has been reported that microRNA-21 can promote osteogenic differentiation of human umbilical cord stem cells (23). Based on these studies, we believe that microRNAs also play a role in the osteogenic differentiation of BMSCs. However, no studies have reported the changes in thr expression of microRNA-21 in bone tissue and serum of OP patients and the effects on osteogenic differentiation of rat BMSCs.

Our results suggest that the expression of microRNA-21 in bone tissue and serum is lower in osteoporotic patients than in healthy controls. In addition, we extracted and isolated, purified BMSCs from SD rats, and transfected microRNA-21 mimics, inhibitors and their corresponding NCs into the cells, and induced osteogenic differentiation of BMSCs by osteogenic induction for 10 days. After transfection of microRNA-21 mimics, ARS and ALP staining showed a significant increase in the number of mineralized nodules, stained calcified area, depth of color, and the expression levels of ALP, Runx2, OCN, and BMP2 genes related to osteogenic differentiation, indicating that microRNA-21 has the potential to promote the differentiation of BMSCs into osteoblasts. After transfection with microRNA-21 inhibitor, osteogenic differentiation ability of BMSCs was decreased, and the ARS and ALP staining showed that the number of mineralized nodules, the area of calcified area stained, and the degree of color depth were significantly reduced. Besides that, the expression levels of ALP, Runx2, OCN, and BMP2 genes related to osteogenic differentiation also decreased, indicating that microRNA-21 inhibitor can inhibit the differentiation of BMSCs into osteoblasts. These results indicate that microRNA-21 has the potential to promote the differentiation of BMSCs into osteoblasts.

In summary, microRNA-21 is expressed at low level in patients with OP, and microRNA-21 can promote the differentiation of BMSCs into osteogenic cells. MicroRNA-21 may serve as a potential therapeutic target for OP.

\section{Acknowledgements}

Not applicable.

Funding

No funding was received.

\section{Availability of data and materials}

The datasets used and/or analyzed during the present study are available from the corresponding author on reasonable request.

\section{Authors' contributions}

ZZ, XL, DZ, YL, ST and ZD drafted the manuscript. ZZ was responsible for the collection of the clinical samples and microRNA detection. XL, DZ and YL were mainly responsible for the cell culture and transfection. XL, DZ, ST and ZD assisted with osteogenic differentiation of rat BMSCs. All authors read and approved the final manuscript.

\section{Ethics approval and consent to participate}

The study was approved by the Ethics Committee of the Second Affiliated Hospital of Qiqihar Medical University (Qiqihar, China). Signed informed consents were obtained from the patients or the guardians.

\section{Patient consent for publication}

Not applicable.

\section{Competing interests}

The authors declare that they have no competing interests. 


\section{References}

1. Anagnostis P, Vakalopoulou S, Christoulas D, Paschou SA, Papatheodorou A, Garipidou V, Kokkoris P and Terpos E: The role of sclerostin/dickkopf-1 and receptor activator of nuclear factor $\mathrm{\kappa B}$ ligand/osteoprotegerin signalling pathways in the development of osteoporosis in patients with haemophilia A and B: A cross-sectional study. Haemophilia 24: 316-322, 2018.

2. Armstrong DJ: Comment on: Patients' preferences for anti-osteoporosis drug treatment: A cross-European discrete choice experiment. Rheumatology (Oxford) 57: 583-584, 2018.

3. Paccou $\mathrm{J}$ and Cortet B: Bisphosphonate drug holidays in postmenopausal osteoporosis: Effect on clinical fracture risk. Response to comments by Bredemeier. Osteoporos Int 29: 521, 2018.

4. Hiligsmann M, Dellaert BG, Watson V and Boonen A: Comment on: Patients' preferences for anti-osteoporosis drug treatment: A cross-European discrete choice experiment: Reply. Rheumatology (Oxford) 57: 584-585, 2018

5. Nogués X, Nolla JM, Casado E, Jódar E, Muñoz-Torres M, Quesada-Gómez JM, Canals L, Balcells M and Lizán L: Spanish consensus on treat to target for osteoporosis. Osteoporos Int 29: 489-499, 2018.

6. Cano A, Chedraui P, Goulis DG, Lopes P, Mishra G, Mueck A, Senturk LM, Simoncini T, Stevenson JC, Stute P, et al: Calcium in the prevention of postmenopausal osteoporosis: EMAS clinical guide. Maturitas 107: 7-12, 2018.

7. Li NB, Sun SJ, Bai HY, Xiao GY, Xu WH, Zhao JH, Chen X, Lu YP and Zhang YL: Preparation of well-distributed titania nanopillar arrays on Ti6Al4V surface by induction heating for enhancing osteogenic differentiation of stem cells. Nanotechnology 29: 045101, 2018.

8. Tian Y, Cui LH, Xiang SY, Xu WX, Chen DC, Fu R, Zhou CL, Liu XQ, Wang YF and Wang XT: Osteoblast-oriented differentiation of BMSCs by co-culturing with composite scaffolds constructed using silicon-substituted calcium phosphate, autogenous fine particulate bone powder and alginate in vitro. Oncotarget 8: 88308-88319, 2017.

9. McAlinden A and Im GI: MicroRNAs in orthopaedic research: Disease associations, potential therapeutic applications, and perspectives. J Orthop Res 36: 33-51, 2018.

10. Raghuwanshi S, Gutti U, Kandi R and Gutti RK: MicroRNA-9 promotes cell proliferation by regulating RUNX1 expression in human megakaryocyte development. Cell Prolif 51: 51, 2018.

11. Wu DM, Zhang YT, Lu J and Zheng YL: Effects of microRNA-129 and its target gene c-Fos on proliferation and apoptosis of hippocampal neurons in rats with epilepsy via the MAPK signaling pathway. J Cell Physiol 233: 6632-6643, 2018.

12. Tokar T, Pastrello C, Rossos AEM, Abovsky M, Hauschild AC, Tsay M, Lu R and Jurisica I: mirDIP 4.1-integrative database of human microRNA target predictions. Nucleic Acids Res 46 (D1): D360-D370, 2018.
13. Livak KJ and Schmittgen TD: Analysis of relative gene expression data using real-time quantitative PCR and the 2(-Delta Delta C(T)) method. Methods 25: 402-408, 2001.

14. Qi M, Zhang L, Ma Y, Shuai Y, Li L, Luo K, Liu W and Jin Y: Autophagy maintains the function of bone marrow mesenchymal stem cells to prevent estrogen deficiency-induced osteoporosis. Theranostics 7: 4498-4516, 2017.

15. Hu Y, Tan LJ, Chen XD, Liu Z, Min SS, Zeng Q, Shen H and Deng HW: Identification of novel potentially pleiotropic variants associated with osteoporosis and obesity using the cFDR method. J Clin Endocrinol Metab 103: 125-138, 2018.

16. Collison J: Osteoporosis: Teriparatide preferable for fracture prevention. Nat Rev Rheumatol 14: 4, 2018.

17. Fukushima H, Shimizu K, Watahiki A, Hoshikawa S, Kosho T, Oba D, Sakano S, Arakaki M, Yamada A, Nagashima K, et al: NOTCH2 Hajdu-Cheney mutations escape SCFFBW7-dependent proteolysis to promote osteoporosis. Mol Cell 68: 645-658.e5, 2017.

18. Xu TB, Li L, Luo XD and Lin H: BMSCs protect against liver injury via suppressing hepatocyte apoptosis and activating TGF- $\beta 1 /$ Bax singling pathway. Biomed Pharmacother 96: 1395-1402, 2017.

19. Chen L, Lu FB, Chen DZ, Wu JL, Hu ED, Xu LM, Zheng MH, Li H, Huang Y, Jin XY, et al: BMSCs-derived miR-223-containing exosomes contribute to liver protection in experimental autoimmune hepatitis. Mol Immunol 93: 38-46, 2018.

20. Wang Y, Luo S, Zhang D, Qu X and Tan Y: Sika pilose antler type I collagen promotes BMSC differentiation via the ERK1/2 and p38-MAPK signal pathways. Pharm Biol 55: 2196-2204, 2017.

21. Huang J, Meng Y, Liu Y, Chen Y, Yang H, Chen D, Shi J and Guo Y: MicroRNA-320a regulates the osteogenic differentiation of human bone marrow-derived mesenchymal stem cells by targeting HOXA10. Cell Physiol Biochem 38: 40-48, 2016.

22. Kim YJ, Bae SW, Yu SS, Bae YC and Jung JS: miR-196a regulates proliferation and osteogenic differentiation in mesenchymal stem cells derived from human adipose tissue. J Bone Miner Res 24: 816-825, 2009.

23. Meng YB, Li X, Li ZY, Zhao J, Yuan XB, Ren Y, Cui ZD, Liu YD and Yang XJ: microRNA-21 promotes osteogenic differentiation of mesenchymal stem cells by the PI3K/ $\beta$-catenin pathway. J Orthop Res 33: 957-964, 2015.

This work is licensed under a Creative Commons Attribution-NonCommercial-NoDerivatives 4.0 International (CC BY-NC-ND 4.0) License. 